\title{
Radiofrequency Ablation in Frequent Ventricular Ectopy
}

\author{
Saurabh Mehrotra ${ }^{1 *}$, Rakesh Sharma ${ }^{2}$ and Sundeep Mishra ${ }^{3}$ \\ ${ }^{1}$ Department of Cardiology, Postgraduate Institute of Medical Education and Research, India \\ ${ }^{2}$ Senior Resident, Postgraduate Institute of Medical Education and Research, India \\ ${ }^{3}$ Department of Cardiology, All India Institutes of Medical Sciences, India
}

Submission: August 16, 2017; Published: October 23, 2017

*Corresponding author: Saurabh Mehrotra, Associate Professor, Department of Cardiology, Postgraduate Institute of Medical Education and Research, Chandigarh, India, Email: rhythm_divine46@yahoo.com

Abstract

Ventricular premature beats (VPBs) are common findings and occur in broad spectrum of population including subjects with structurally normal hearts and those with cardiac disease, independent of severity. Most of these arise from right ventricular outflow tract and may be asymptomatic or may cause variety of symptoms. High VPB burden is associated with tachycardia induced cardiomyopathy. Symptomatic patients and patients with cardiomyopathy attributable to high VPB burden are offered drugs or catheter ablation. Catheter ablation has high success and low complication rate. Till date VPB frequency and the time point when ablation is recommended is still not clear.

\section{Introduction}

Ventricular premature beats (VPBs), also referred to as ventricular premature complexes are early depolarization's of ventricular myocardium arising in a variety of situations. VPCs/NSVT are common and occur in a broad spectrum of the population including patients without structural heart disease and those with any form of cardiac disease, independent of severity [1]. The incidence of VPBs in subjects with structurally normal hearts varies according to observational studies. Incidence of PVBs was 7.8\% in participants during evaluation of 12-lead ECGs in a large healthy military population, with a much lower incidence in age group below 20 (4.6\%) compared to those older than 50 years of age (21.7\%) [2]. Hinkle et al. [3] reported that the incidence of asymptomatic ventricular arrhythmias was $62 \%$ in a mixed population of healthy individuals and patients with known heart disease.

Ventricular arrhythmias occurring in structurally normal hearts are labelled as idiopathic ventricular arrhythmias (VA) and accounts for approximately $10 \%$ of the patients with ventricular tachycardia (VT) [4]. Outflow tract arrhythmias are the most common type of idiopathic VA and more than $70-80 \%$ of idiopathic VTs or VPBs arise from right ventricular (RV) OT. Prognostic implication of VPCs may vary depending on underlying heart disease, left ventricular functions, age of patient and associated co-morbidities. Prognosis is usually favourable in patients with structurally normal heart and age below 30 years.
Also frequent isolated ectopic beats, mostly originating from the right ventricular outflow tract have been reported as a cause of tachycardiomyopathy, a reversible form of congestive heart disease that resolves after elimination of the culprit arrhythmia either by medical treatment or by Radiofrequency Ablation (RFA). As most of VA arise from RVOT so our further discussion will primarily focus on ventricular arrhythmias arising from right ventricular outflow tract.

\section{Prevalence}

The prevalence of VPBs is directly related to the study population, method of detection and duration of observation. In patients with no known heart disease, VPBs occur in approximately 1 percent of routine 12-lead ECG of 30 to 60 seconds duration $[2,4]$. When 24-hour ambulatory monitoring is used, up to 80 percent of apparently healthy people have occasional VPBs [5,6]. The frequency of VPBs increases with increasing age and also in patients with underlying heart disease. There is an age-related increase in the prevalence of VPBs in normal individuals and those with underlying heart disease $[2,6,7]$. The occurrence of frequent VPBs accounting for more than 20 percent of overall heart beats is rare, seen in less than 2 percent of patients [8].

\section{Symptoms}

Ventricular ectopic activity is commonly encountered in clinical practice. Usually, it is not associated with life-threatening 
consequences in the absence of significant structural heart disease. However, frequent ventricular ectopic beats can be extremely symptomatic and even incapacitating in some patients because of palpitations or dizziness. VPBs rarely cause hemodynamic instability, except when associated with severe left ventricular systolic dysfunction or when occurring with bradycardia.

VPBs are associated with several characteristic findings on history; physical examination and electrocardiogram. Symptoms variously reported include palpitations, pounding sensation in the neck, dyspnoea, dizziness, pre- syncope, syncope, reduced exercise capacity and decreased quality of life. Other symptoms described by patients may include coughing, claudication and dysphagia.

Increased VPB/ NSVT burden may cause tachycardia induced cardiomyopathy with heart failure symptoms. Cardiomyopathy induced by atrial tachyarrhythmias has been well described [9-12]. Although the mechanism for tachycardia-induced cardiomyopathy secondary to atrial tachyarrhythmias with fast ventricular activation is well understood, the mechanisms for PVB- induced cardiomyopathy are less clear. Besides LV dyssynchrony due to LBBB during PVBs, other causes such as increased oxygen consumption [13] have been implicated. Furthermore, the so-called apical-to-basal "squeezing effect" in systole during physiological activation of the LV is disrupted during RVOT PVCs with LBBB morphology, which may further impair LV systolic output [14]. Till date the important question that how much PVB burden is required to cause PVB-induced cardiomyopathy is not yet completely answered. Whereas in one study from Carbeillera Pol et al. [15] the PVB burden was not associated with the development of cardiomyopathy (PVB burden $>10 \%$ was used as inclusion criteria in this study), several other studies showed correlation between PVB frequency and cardiomyopathy. Most of electrophysiologist consider $>20 \%$ VPBs burden as criteria to label as tachycardia induced cardiomyopathy in setting of frequent ventricular ectopics and associated left ventricular systolic dysfunction.

In addition to the overall frequency of VPBs, QRS duration as well as epicardial site of origin of VPBs appears to play a role in the development of cardiomyopathy and are associated with outcomes following catheter ablation. Wider QRS complexes appear more likely to result in cardiomyopathy with a lower overall burden of VPBs while also being associated with longer times to normalization of $\mathrm{LV}$ systolic function following ablation, while epicardial VPB origin also appears to predict delayed LV function recovery.

The incidence of PVB-induced cardiomyopathy is higher in older patients [16] and the pathophysiology is less well understood. It is often more difficult to determine whether PVBs are a result of reduced LV function or the cause. It is well described that PVBs/NSVTs are a common finding on 24- hour holter recordings in patients with ischemic or dilated cardiomyopathy. The indication for primary ICD implantation in these patients to reduce the arrhythmogenic risk for SCD is dependent on LV function and not arrhythmia burden. The increased risk for SCD is present even if no arrhythmias have been documented.

\section{How to Diagnose Origin of VPBs}

Detailed intracardiac electrical mapping has demonstrated that the vast majority of outflow tract VPBs/VTs originate from the anterior and superior septal aspect of the right ventricular outflow tract (RVOT), just inferior to the pulmonic valve $[17,18]$ Less commonly, the site of origin can be localized to the right ventricular (RV) infundibulum, RV free wall, and posterior aspect of the interventricular septum. In approximately $10 \%$ to $15 \%$ of cases, the arrhythmia originates from the left ventricular outflow tract (LVOT) and can be mapped to the region of the aortic cusps $[19,20](1,8-10)$. Rarely, outflow tract VPCs/VTs can be ablated from within the anterior inter ventricular vein, aortomitral continuity, or the root of the pulmonary artery.

Lead V2 transition ratio can be used for distinguishing LVOT from RVOT origin in patients with left bundle branch pattern idiopathic PVBs with lead V3 precordial transition. This measure accounts for variations in body habitus, cardiac rotation, respiratory variation, and ECG lead positioning by measuring precordial transition during the PVB/VT relative to the SR precordial transition. A V2 transition ratio 0.6 predicted an LVOT origin with $95 \%$ sensitivity and $100 \%$ specificity. For patients referred for catheter ablation of OTVT, this simple ECG measurement might be performed in the office both to help plan an ablation strategy and to enhance patient counselling with regard to procedural time, potential outcome, and risks associated with arterial access, mapping, and ablation. One might argue that the V2 transition ratio is cumbersome for everyday use in clinical practice. In the electrophysiology lab, this measurement is easily made with digital calipers available on any clinical electrophysiologic recording system. For more practical clinical use a precordial transition during the PVB/ VT that occurs later than the SR transition excludes an LVOT origin with $100 \%$ accuracy. This simple measure can be easily used by any cardiologist or electrophysiologist when counselling patients about PVB ablation. For more practical clinical use, precordial transition during the PVB/VT that occurs later than the sinus rhythm transition excludes an LVOT origin with $100 \%$ accuracy. This simple measure can be easily used by any cardiologist or electrophysiologist when counselling patients about PVB ablation.

In case of RVOT PVBs, 12-lead surface ECG is crucial for identifying the origin of PVBs/VTs from this anatomically complex region [21]. Resting 12- lead ECGs in sinus rhythm are usually normal, with up to $10 \%$ of patients presenting with incomplete or even complete right bundle branch block (RBBB). 
Typically, PVBs originating from the RVOT have an inferior axis with left bundle branch block (LBBB) morphology, and a late R/S transition at V4 in the precordial leads. A QRS duration $<140 \mathrm{~ms}$ is suggestive of a PVB with a 'septal' origin, whereas a QRS duration $>140 \mathrm{~ms}$ favours a 'free wall' origin, particularly when notches are seen in the down stroke of the QRS of the inferior leads [22].

\section{Diagnostic Evaluation}

The diagnostic evaluation of patients with symptoms suggesting VPBs includes an electrocardiogram (ECG) or ambulatory cardiac monitoring, if VPBs are not recorded in ECG. Also 24 hour holter monitoring is best accepted approach to quantifying the frequency of PVBs as a percentage of total heart beats and determine if they are monomorphic or multimorphic.

Echocardiography should be performed focusing on the presence or absence of underlying structural heart disease and ventricular systolic functions. It is important to distinguish the benign VPBs originating in the right ventricular outflow tract from those related to arrhythmogenic right ventricular cardiomyopathy, as the VPB morphology may be quite similar but the prognosis entirely different. Catheter ablation can be quite an effective treatment in the first case, while patients with the last condition often need an implantable cardioverterdefibrillator as protection from sudden cardiac death. ARVC should generally be suspected in patients with a family history of sudden death and/or $\mathrm{T}$ wave inversion in the right precordial leads.

Exercise treadmill stress test to evaluate the response of the VPBs to exercise, determine the VPB morphology, determine if sustained or non sustained ventricular tachycardia (VT) can be induced with exercise, as well as to screen for underlying ischemia. Catecholamine-sensitive VPBs may increase during exercise, as well as those related to ischemia; more commonly, however, VPBs are suppressed during exercise and remerge during recovery phase. Catecholamine or exercise-induced VPBs respond well to beta blocker therapy.

Correctable causes or triggers should be sought by clinical history (inquiring about possible underlying cardiovascular diseases, but also about use of alcohol or caffeine-containing beverages, or illicit drugs, etc) and/or laboratory testing (electrolyte levels, thyroid stimulating hormone [TSH]). For documented nocturnal VPBs, sleep apnea needs to be considered and polysomnography performed, when indicated. Further testing is indicated only when this initial evaluation identifies significant abnormalities that require further evaluation.

\section{Treatment}

In persons with frequent VPBs, evaluation and management is based on symptomatic status and presence or absence of underlying structural heart disease which has prognostic significance and may require specific therapy. There is no clear evidence that VPB suppression with beta blockers or antiarrhythmic drugs improves overall survival in patients who have no symptoms and have not had a major arrhythmic event. Thus, the only indications for the use of beta blockers or antiarrhythmic drugs for VPB suppression are for symptomatic patients or for patients with cardiomyopathy felt to be possibly related to frequent VPBs.

According to the current guidelines for the management of symptomatic PVCs [23], beta-blockers are the drug of choice. However, the efficacy of beta-blocker therapy (namely atenolol and metoprolol) is generally modest, with a reduction of PVC burden between 10-25\% [24,25]. The efficacy of calcium channel antagonists such as verapamil varies in several reports, with reasonable efficacy in patients with idiopathic VT [26-28] but it is less effective in patients with only PVCs, most likely as a result of different underlying mechanisms [25]. Following these, Class I antiarrhythmic drugs such as propafenone or flecainide are recommended. Although treatment with antiarrhythmic drugs in symptomatic patients with structurally normal hearts may be reasonable [23], they are contraindicated in patients with cardiomyopathy due to their proarrhythmic effects [29]. In these patients, beta-blockers and amiodarone are the only antiarrhythmic drugs available. However, due to the frequent and significant side effects associated with amiodarone [30], it should only be administered in patients refusing catheter ablation or after failed catheter ablation. Since catheter ablation of RVOT PVB/VTs is a highly effective therapy with a very low complication rate, this should be the treatment of choice.

Patients found to have underlying structural heart disease will typically receive medical therapy specific to their disease process. In many cases, this therapy will also reduce the frequency of VPBs. Examples of therapy which can reduce the frequency of VPBs are beta blockers, which improve survival in patients with a prior myocardial infarction or heart failure, and antihypertensive therapy, which may induce regression of $\mathrm{LVH}$ in patients with hypertension.

For patients with symptomatic VPBs in whom beta blockers or calcium channel blockers have not resulted in symptomatic improvement, additional therapeutic options include antiarrhythmic medication and radiofrequency catheter ablation. For most patients, either approach is a reasonable first choice. However, for patients with frequent VPBs associated with left ventricular dysfunction, radiofrequency ablation is preferred modality. In patients with high PVCs burden ( $>20 \%$ PVCs $/ 24$ hours) is associated with increased risk of developing left ventricular dysfunction so prophylactic ablation may be proposed even when patients are asymptomatic.

\section{Outcomes of Radiofrequency Ablation}

In general, the success rate of catheter ablation of arrhythmias originating from the RVOT is reported to be high [24]. In most studies, the acute success rate is reported to 
be $>80 \%$ [23]. Also after successful ablation the recurrence rate is generally not exceeding $5 \%$ even after long-term follow-up [23,31-35]. Predictors of lower success rate of catheter ablation are only infrequent PVCs or non-inducibility of the clinical arrhythmia during the procedure. The pathophysiologic basis for idiopathic PVCs, NSVTs and ventricular tachycardias vary, but are considered to be mostly related to triggered activity. Therefore, an isoproterenol infusion may be required to induce these arrhythmias during an electrophysiology study [26,36]. Failure to induce arrhythmia even after isoproterenol administration may decrease the success rate of catheter ablation due to the lack of a clear endpoint. When there is non-inducibility of the clinical VT, ablation of monomorphic PVCs with the same morphology as the documented arrhythmia to eliminate the arrhythmia trigger may be a reasonable strategy. In addition to activation mapping, pace-mapping may be performed. Non-contact mapping can be considered in difficult cases to identify the PVC origin even in patients with rare PVCs [37-39]. As deep sedation is considered by some investigators to reduce the spontaneous PVC burden, reducing the amount of sedation and analgesia can often reduce suppression of PVCs and increase their frequency.

In general, catheter ablation of idiopathic PVCs originating from either the RVOT or left-sided structures is usually considered to be safe [40]. Zhong et al. [41] recently published a procedure-related complication rate of $5.6 \%$ (12 patients). The use of intracardiac echocardiography may help to better de ne the anatomy in complex cases during ablation of RVOT arrhythmias and has been previously shown to be helpful, particularly during ablation of tachycardias from the LVOT. Although RF energy is still the gold standard for ablation within the RVOT [23,32-35,40] with high efficacy and safety [40], cryoablation is a reasonable alternative that allows for almost pain-free ablation [42].

\section{Conclusion}

VPBs are common finding in absence or presence of heart diseases and also person may be asymptomatic or symptomatic. High VPB burden may lead to tachycardia induced cardiomyopathy and severity of ventricular dysfunction has been correlated with VPB frequency. Catheter ablation is an excellent option with $>80 \%$ success rate for the treatment of RVOT PVC/VTs and is superior to antiarrhythmic drug treatment Even during long-term follow-up significant complications are rare with radiofrequency ablation. Therefore radiofrequency ablation may be offered to all patients with symptomatic RVOT PVC/VT refractory to antiarrhythmic drug treatment or when antiarrhythmic drugs are not desired by the patient regardless of the PVC burden. In patients with asymptomatic RVOT PVCs and preserved LVEF, catheter ablation should be considered if the arrhythmia burden is $>20 \%$ to prevent the development of tachycardia-induced cardiomyopathy. In patients with reduced LVEF suspected to be due to frequent VPBs, catheter ablation should be considered unless contraindicated.

\section{References}

1. Kostis JB, McCrone K, Moreyra AE, Gotzoyannis S, Aglitz NM, et al. (1981) Premature ventricular complexes in the absence of identifiable heart disease. Circulation. 63(6): 1351-1356.

2. Hiss RG, Lamb LE (1962) Electrocardiographic findings in 122,043 individuals. Circulation 25: 947-961.

3. Hinkle LE, Carver ST, Stevens M (1969) The frequency of asymptomatic disturbances of cardiac rhythm and conduction in middle-aged men. Am J Cardiol 24(5): 629-650.

4. Basiouny T, Kholeif HAE-L, El-Tahan MH, Karim M, Attia W, et al. (2014) Radiofrequency catheter ablation of premature ventricular complexes from right ventricular outflow tract in patients with left ventricular dilation and/or dysfunction. Egypt Heart J 66(4): 351-361.

5. Sobotka PA, Mayer JH, Bauernfeind RA, Kanakis C, Rosen KM (1981) Arrhythmias documented by 24-hour continuous ambulatory electrocardiographic monitoring in young women without apparent heart disease. Am Heart J 101(6): 753-759.

6. Brodsky M, Wu D, Denes P, Kanakis C, Rosen KM (1977) Arrhythmias documented by 24 hour continuous electrocardiographic monitoring in 50 male medical students without apparent heart disease. Am J Cardiol 39(3): 390-395

7. Simpson RJ, Cascio WE, Schreiner PJ, Crow RS, Rautaharju PM, et al. (2002) Prevalence of premature ventricular contractions in a population of African American and white men and women: the Atherosclerosis Risk in Communities (ARIC) study. Am Heart J 143(3): 535-540.

8. Yang J, Dudum R, Mandyam MC, Marcus GM (2014) Characteristics of unselected high-burden premature ventricular contraction patients. Pacing Clin Electrophysiol 37(12): 1671-1680.

9. Noda T, Shimizu W, Taguchi A, Aiba T, Satomi K, et al. (2005) Malignant entity of idiopathic ventricular fibrillation and polymorphic ventricular tachycardia initiated by premature extrasystoles originating from the right ventricular outflow tract. J Am Coll Cardiol 46(7): 1288-1294.

10. Viskin S, Rosso R, Rogowski O, Belhassen B (2005) The "shortcoupled" variant of right ventricular outflow ventricular tachycardia: a not-so-benign form of benign ventricular tachycardia? J Cardiovasc Electrophysiol 16(8): 912-916.

11. Grogan M, Smith HC, Gersh BJ, Wood DL (1992) Left ventricular dysfunction due to atrial fibrillation in patients initially believed to have idiopathic dilated cardiomyopathy. Am J Cardiol 69(19): 15701573.

12. Shinbane JS, Wood MA, Jensen DN, Ellenbogen KA, Fitzpatrick AP, et al. (1997) Tachycardia-induced cardiomyopathy: a review of animal models and clinical studies. J Am Coll Cardiol 29(4): 709-715.

13. Chardack WM, Gage AA, Dean DC (1965) Paired and coupled electrical stimulation of the heart. Bull N Y Acad Med 41: 462-480.

14. Shiraishi H, Ishibashi K, Urao N, Tsukamoto M, Hyogo M, et al. (2002) A case of cardiomyopathy induced by premature ventricular complexes. Circ J 66(11): 1065-1067.

15. Carballeira Pol L, Deyell MW, Frankel DS, Benhayon D, Squara F, et al. (2014) Ventricular premature depolarization QRS duration as a new marker of risk for the development of ventricular premature depolarization-induced cardiomyopathy. Heart Rhythm 11(2): 299306.

16. Yarlagadda RK, Iwai S, Stein KM, Markowitz SM, Shah BK, et al. (2005) Reversal of cardiomyopathy in patients with repetitive monomorphic ventricular ectopy originating from the right ventricular outflow tract. Circulation 112(8): 1092-1097. 
17. Movsowitz C, Schwartzman D, Callans DJ, Preminger M, Zado E, et al. (1996) Idiopathic right ventricular outflow tract tachycardia: narrowing the anatomic location for successful ablation. Am Heart J 131(5): 930-936.

18. Latif S, Dixit S, Callans DJ (2008) Ventricular arrhythmias in normal hearts. Cardiol Clin 26(3): 367-380.

19. Callans DJ, Menz V, Schwartzman D, Gottlieb CD, Marchlinski FE (1997) Repetitive monomorphic tachycardia from the left ventricular outflow tract: electrocardiographic patterns consistent with a left ventricular site of origin. J Am Coll Cardiol 29(5): 1023-1027.

20. Bala R, Garcia FC, Hutchinson MD, Gerstenfeld EP, Dhruvakumar S, et al. (2010) Electrocardiographic and electrophysiologic features of ventricular arrhythmias originating from the right/left coronary cusp commissure. Heart Rhythm 7(3): 312-322.

21. Buxton AE (1997) Nonsustained Ventricular Tachycardia Update: Epidemiology, Pathophysiology, Immediate Evaluation and Management, Long-Term Management, Experimental and Theoretical Developments. Card Electrophysiol Rev 1(1-2): 92-96.

22. Dixit S, Gerstenfeld EP, Callans DJ, Marchlinski FE (2003) Electrocardiographic patterns of superior right ventricular outflow tract tachycardias: distinguishing septal and free-wall sites of origin. J Cardiovasc Electrophysiol 14(1): 1-7.

23. Aliot EM, Stevenson WG, Garrote JMA, Bogun F, Calkins CH, et al. (2009) EHRA/HRS Expert Consensus on Catheter Ablation of Ventricular Arrhythmias: developed in a partnership with the European Heart Rhythm Association (EHRA), a Registered Branch of the European Society of Cardiology (ESC), and the Heart Rhythm Society (HRS); in collaboration with the American College of Cardiology (ACC) and the American Heart Association (AHA). Europace 11(6): 771-817.

24. Krittayaphong R, Bhuripanyo K, Punlee K, Kangkagate C, Chaithiraphan S (2002) Effect of atenolol on symptomatic ventricular arrhythmia without structural heart disease: a randomized placebo-controlled study. Am Heart J 144(6): e10.

25. Stec S, Sikorska A, Zaborska B, Kryński T, Szymot J, et al. (2012) Benign symptomatic premature ventricular complexes: short- and long-term efficacy of antiarrhythmic drugs and radiofrequency ablation. Kardiol Pol 70(4): 351-358.

26. Farzaneh-Far A, Lerman BB (2005) Idiopathic ventricular outflow tract tachycardia. Heart 91(2): 136-138.

27. Niwano S, Wakisaka Y, Niwano H, Fukaya H, Kurokawa S, et al. (2009) Prognostic significance of frequent premature ventricular contractions originating from the ventricular outflow tract in patients with normal left ventricular function. Heart 95(15): 1230-1237.

28. European Heart Rhythm Association, Heart Rhythm Society, Zipes DP, Camm AJ, Borggrefe M, et al. (2006) ACC/AHA/ESC 2006 guidelines for management of patients with ventricular arrhythmias and the prevention of sudden cardiac death: a report of the American College of Cardiology/American Heart Association Task Force and the European Society of Cardiology Committee for Practice Guidelines (Writing Committee to Develop Guidelines for Management of Patients With Ventricular Arrhythmias and the Prevention of Sudden Cardiac Death). J Am Coll Cardiol 48(5): e247-e346.
29. Investigators TCAST (CAST) (1989) Preliminary Report: Effect of Encainide and Flecainide on Mortality in a Randomized Trial of Arrhythmia Suppression after Myocardial Infarction. N Engl J Med 321(6): 406-412.

30. Singh BN (1999) Current antiarrhythmic drugs: an overview of mechanisms of action and potential clinical utility. J Cardiovasc Electrophysiol 10(2): 283-301.

31. Rillig A, Lin T, Ouyang F, Kuck KH, Tilz RR (2014) Which Is The Appropriate Arrhythmia Burden To Offer RF Ablation For RVOT Tachycardias? J Atr Fibrillation 7(4):1157.

32. Ouyang F, Fotuhi P, Ho SY, Hebe J, Volkmer M, et al. (2002) Repetitive monomorphic ventricular tachycardia originating from the aortic sinus cusp: electrocardiographic characterization for guiding catheter ablation. J Am Coll Cardiol 39(3): 500-508.

33. Vohra J, Shah A, Hua W, Gerloff J, Riters A (1996) Radiofrequency ablation of idiopathic ventricular tachycardia. Aust N Z J Med 26(2): 186-194.

34. Talwar KK, Singh B, Goel P, Saxena A, Bahl VK, et al. (1996) Radiofrequency ablation of idiopathic ventricular tachycardia. Indian Heart J 48: 49-52.

35. Rodriguez LM, Smeets JL, Timmermans C, Wellens HJ (1997) Predictors for successful ablation of right- and left-sided idiopathic ventricular tachycardia. Am J Cardiol 79(3): 309-314.

36. Iwai S, Cantillon DJ, Kim RJ, Markowitz SM, Mittal S, et al. (2006) Right and left ventricular outflow tract tachycardias: evidence for a common electrophysiologic mechanism. J Cardiovasc Electrophysiol 17(10): 1052-1058.

37.Zhang F, Yang B, Chen H, Ju W, Kojodjojo P, et al. (2013) Magnetic versus manual catheter navigation for mapping and ablation of right ventricular outflow tract ventricular arrhythmias: a randomized controlled study. Heart Rhythm 10(8): 1178-1183.

38. Schneider HE, Kriebel T, Jung K, Gravenhorst VD, Paul T (2010) Catheter ablation of idiopathic left and right ventricular tachycardias in the pediatric population using noncontact mapping. Heart Rhythm $7(6): 731-739$.

39. Friedman PA, Asirvatham SJ, Grice S, Glikson M, Munger TM, et al. (2002) Noncontact mapping to guide ablation of right ventricular outflow tract tachycardia. J Am Coll Cardiol 39(11): 1808-1812.

40. Rillig A, Meyerfeldt U, Birkemeyer R, Treusch F, Kunze M, et al. (2008) Catheter ablation within the sinus of Valsalva--a safe and effective approach for treatment of atrial and ventricular tachycardias. Heart Rhythm 5(9): 1265-1272.

41. Zhong L, Lee YH, Huang XM, Asirvatham SJ, Shen WK, et al. (2014) Relative efficacy of catheter ablation vs antiarrhythmic drugs in treating premature ventricular contractions: a single-center retrospective study. Heart Rhythm 11(2): 187-193.

42. Kennedy HL, Whitlock JA, Sprague MK, Kennedy LJ, Buckingham TA, et al. (1985) Long-term follow-up of asymptomatic healthy subjects with frequent and complex ventricular ectopy. N Engl J Med 312(4): 193-197. 
(C) (i) This work is licensed under Creative DOI: 10.19080/JOJCS.2017.04.555643
Your next submission with Juniper Publishers will reach you the below assets

- Quality Editorial service

- Swift Peer Review

- Reprints availability

- E-prints Service

- Manuscript Podcast for convenient understanding

- Global attainment for your research

- Manuscript accessibility in different formats ( Pdf, E-pub, Full Text, Audio)

- Unceasing customer service

Track the below URL for one-step submission https://juniperpublishers.com/online-submission.php 